\title{
Mechanical, Thermal and Electromagnetic Shielding Effectiveness of MWCN-ABS Films
}

\author{
R. B. Jagadeesh Chandra ${ }^{1}$ - B. Shivamurthy ${ }^{2}$ (D) M. Sathish Kumar ${ }^{1}$ - B. H. S. Thimmappa ${ }^{3}$
}

Received: 26 February 2021 / Revised: 1 May 2021 / Accepted: 11 May 2021 / Published online: 1 June 2021

(c) The Author(s) 2021

\begin{abstract}
The multi-walled carbon nanotubes (MWCNTs) and the poly(acrylonitrile-co-butadiene-co-styrene) (ABS) granulates are dispersed in acetone separately using a magnetic stirrer followed by ultrasonication. Further, both the solutions were mixed with magnetic stirring followed by ultrasonication. Neat-ABS film, $0.25 \mathrm{wt} \%, 0.5 \mathrm{wt} \%$ and $1 \mathrm{wt} \%$ of MWCNT-ABS nanocomposite films of the average thickness of $140 \mu \mathrm{m}$ are fabricated by the solution molding using a petri dish, followed by room temperature curing and further hot compression to maintain uniform thickness. The tensile properties, thermal stability, electrical conductivity, and EMSE of all films are investigated. The results indicate that the addition of MWCNTs to ABS enhanced the mechanical properties and electrical conductivity, thermal stability, and EMSE. The $0.25 \mathrm{wt} \% \mathrm{MWCNT}$-ABS nanocomposite films show attractive mechanical, electrical, thermal, and EMSE as compared to neat-ABS films. More than $0.25 \mathrm{wt} \%$ MWCNTs in the ABS matrix deteriorates the tensile strength. However, $0.5 \mathrm{wt} \%$ MWCNT-ABS nanocomposites exhibit better tensile strength, Young's modulus, electrical conductivity, and EMSE than neat-ABS. In this research, we used a low quantity of MWCNTs and followed a one-time heating process in the entire fabrication, and produced MWCNT-ABS nanocomposite films with reasonable properties. Hence, this may be one of the options to produce nanocomposites suitable for EMS materials. We recommend that these films may be used as interlayers to develop an X-band range electromagnetic wave shielding material.
\end{abstract}

Keywords Electromagnetic shielding effectiveness (EMSE) $\cdot$ Electrical conductivity $\cdot$ MWCNT $\cdot$ High aspect ratio

\section{Introduction}

B. Shivamurthy

shiva.b@manipal.edu

R. B. Jagadeesh Chandra

jagadeesh.chandra@manipal.edu

B. H. S. Thimmappa

bhs.thims@manipal.edu

1 Department of Electronics and Communication Engineering, Manipal Institute of Technology, Manipal Academy of Higher Education, Manipal 576104, India

2 Department of Mechanical and Manufacturing Engineering, Manipal Institute of Technology, Manipal Academy of Higher Education, Manipal 576104, India

3 Department of Chemistry, Manipal Institute of Technology, Manipal Academy of Higher Education, Manipal, Karnataka 576 104, India
Due to information technology's continuous development, wireless communication systems, smart electronic systems, and electronic devices are continuously increased. These systems create electromagnetic (EM) pollution. Hence, it is necessary to protect the environment, electronic instruments, and EM sensitive workspace from electromagnetic interference (EMI) by suitable shielding material. In this regard, developing a useful EMI shielding material becomes an important research in the electronic materials domain [1]. In this context, material researchers focused on developing conductive filler reinforced polymer-based light-weight, corrosion resistant, flexible, and easy to manufacture materials. The EM shielding performance depends upon several important parameters such as fillers shape, quantity and aspect ratio of fillers, dielectric constant, ambient temperature, electrical conductivity, and the thickness of the shielding material $[2,3]$. The polymer nanocomposites with carbon fillers such as carbon nanotubes (CNTs), carbon fiber, 
carbon black (CB), carbon nanofibers, graphene, and graphite have numerous applications in the energy, electronics, automotive, and aerospace industry sectors. These polymer composites are also used as protective covers for EMI shielding and electrostatic discharge applications $[4,5]$. The literature indicates that CNTs filled epoxy composites are found suitable for EMI shielding in the frequency range $10 \mathrm{MHz}$ to $1.5 \mathrm{GHz}$. However, shielding in the range of $8-12 \mathrm{GHz}$ (X-band) is more important for many military and commercial applications [6]. In this context, Schmitz et al. studied electrical conductivity, mechanical properties, and EMSE of ABS-CNT, ABS-CNT/CB, and ABS-CB composites prepared by melt mixing and fused deposition method. They found improved results due to the addition of carbonaceous filler [7, 8]. Also, it was noticed that the high aspect ratio of MWCNTs reinforced polymer composites exhibit better EMSE compared to low aspect ratio fillers. This observation was attributed to the network structure of MWCNTs in the polymer matrix [9-12].

Poly(acrylonitrile-co-butadiene-co-styrene) is an amorphous thermoplastic polymer comprising three monomers acrylonitrile, butadiene, and styrene. The different amounts of each monomer can be added to develop the finished product according to the required properties and surface finish. ABS is tough and highly durable. It is easy to process through injection molding, $3 \mathrm{D}$ printing, and compression molding. This versatility of ABS plastic makes it suitable for many applications. Due to this, many researchers attempted to develop ABS based EM shielding materials by reinforcing fillers. Kuan et al. [13] developed MWCNT-ABS nanocomposites by direct compounding and twice-fabrication method and found improvement of EMSE due to the addition of $2.8 \mathrm{wt} \%$ MWCNTs. Al-Saleh et al. [14] investigated various concentration MWCNT-ABS nanocomposites prepared by the melt-mixing method. They reported the EMSE and electrical conductivity increased with the CNT concentration. The ABS with $3 \mathrm{wt} \%$ CNT nanocomposites of $1.1 \mathrm{~mm}$ thickness records EMSE of $17 \mathrm{~dB}$ in the operating range of 100-1500 MHz. Al-Saleh [15] also developed $1 \mathrm{~mm}$ thick of $10 \mathrm{wt} \%$ CNT-UHMWPE nanocomposite films by solution method and followed by compression molding and found an enhancement of EMSE, and also reported are independent of the frequency in the X-band range. Jyoti et al. [16] reported that the $10 \mathrm{wt} \%$ MWCNT-ABS showed the EMSE up to $-39 \mathrm{~dB}$ in the Ku band. Kapoor et al. [17] reported enhanced static, dynamic mechanical, and thermal properties of MWCNT-ABS nanocomposites compared to neat ABS. It is found that most of the literature reported on CNT reinforced ABS bulk composites. However, very few researchers tried to develop thin films that may be used to make the multi-layer structure, which is very efficient in EM wave absorption. Based on this phenomenon, we attempted primarily to investigate the mechanical, thermal, electrical, and EMSE of high aspect ratio MWCNTs reinforced ABS composite thin films via the solvent method.

\section{Materials and Methods}

The ABS was procured by M/s. Konkan Specialty Polyproducts Pvt. Ltd. India., used as matrix material. The material code is $\mathrm{HI} 121 \mathrm{H}$. High aspect ratio MWCNTs were procured from M/s Ad-Nano Technologies Pvt. Ltd. India. The acetone of purity of $99 \%$ supplied by the M/s Merk was used as a solvent. The basic properties of ABS and MWCNTs used in manufacturing nanocomposite films are presented in Table 1.

In this research work, the MWCNT-ABS nanocomposite films are manufactured by the solution casting, followed by the hot compression molding method. The morphology of the MWCNTs is investigated by field emission scanning electron microscope (FESEM). The tensile properties for the MWCNT-ABS films were tested according to ASTM D882 [18] with a constant crosshead speed. The electrical conductivity of the composite nanofilms is investigated as per ASTM D257 [19]. Thermal behavior is investigated by thermogravimetric analysis, and the EMSE was estimated for individual films by vector network analyzer.
Table 1 Specifications of ABS and MWCNTs

\begin{tabular}{llll}
\hline ABS & & MWCNT & \\
\hline Grade & LG ABS HI 121H & Purity & $99 \%$ \\
Density & $1.05 \mathrm{~g} \mathrm{~cm}^{-3}$ & Outer diameter & $10-30 \mathrm{~nm}$ \\
Melt mass flow rate & $22 \mathrm{~g} / 10 \mathrm{~min}$ & Inner diameter & $5-10 \mathrm{~nm}$ \\
Molding shrinkage & $0.04-0.70 \%$ & Surface area & $110-350 \mathrm{~m}^{2} \mathrm{~g}^{-1}$ \\
Tensile modulus & $2350 \mathrm{Mpa}$ & CNT content & $\sim 95-99 \%$ \\
Tensile strength & $49 \mathrm{Mpa}$ & Bulk density & $0.14 \mathrm{~g} \mathrm{~cm}^{-3}$ \\
Elongation & $5 \%$ & Amorphous carbon & $<1 \%$ \\
Rockwell hardness & 110 & & \\
\hline
\end{tabular}




\section{Results and Discussion}

\subsection{Fabrication of MWCNT-ABS nanocomposite films}

Accurately weighed $0.25 \mathrm{wt} \%$ of MWCNTs using a digital weighing balance up to an accuracy of three decimal places and transferred to a flat bottom conical flask that contains acetone. The MWCNTs were dispersed in acetone by magnetic stirring ( $1 \mathrm{~h}$ at $300 \mathrm{rpm}$ ) followed by ultrasonication. The resultant solution, called solution A. As bought $\mathrm{ABS}$ granules are kept in a hot oven at $60{ }^{\circ} \mathrm{C}$ for $1 \mathrm{~h}$ to remove the material's water molecules and moisture. Then the required quantity of ABS was weighed and dissolved in acetone in another flat-bottom conical flask. The resultant stock solution called B. Now both the solutions were mixed further through magnetic stirring followed by the ultrasonication process. After obtaining a uniform mix of the ABS-MWCNTs solution was poured slowly into a petri dish and kept in an open environment for $24 \mathrm{~h}$ to enable solvent evaporation. The excess solvent is removed by keeping the samples in the vacuum desiccator for about $6 \mathrm{~h}$. Finally, the nanocomposite film was pressed in a hot oven at $90^{\circ} \mathrm{C}$ for a few minutes to obtain uniform thickness and free from surface defects. This process was repeated to prepare nanocomposite films with 0.5 and $1 \mathrm{wt} \%$ MWCNT mix with ABS. It is found that each film of an average thickness of $140 \pm 20 \mu \mathrm{m}$. The photographs of all the stages and sequences of the process are shown in Fig. 1.

\subsection{Morphology}

The morphology of MWCNTs is investigated by using a FESEM. The FESEM image of MWCNTs are shown in Fig. 2. It is confirmed that the MWCNTs used in this study are of high aspect ratio and most suitable to get a network structure in the polymer matrix.

\subsection{Mechanical Properties}

It is reported in the literature that more quantity of functionalized CNT can disperse by the solution mixing

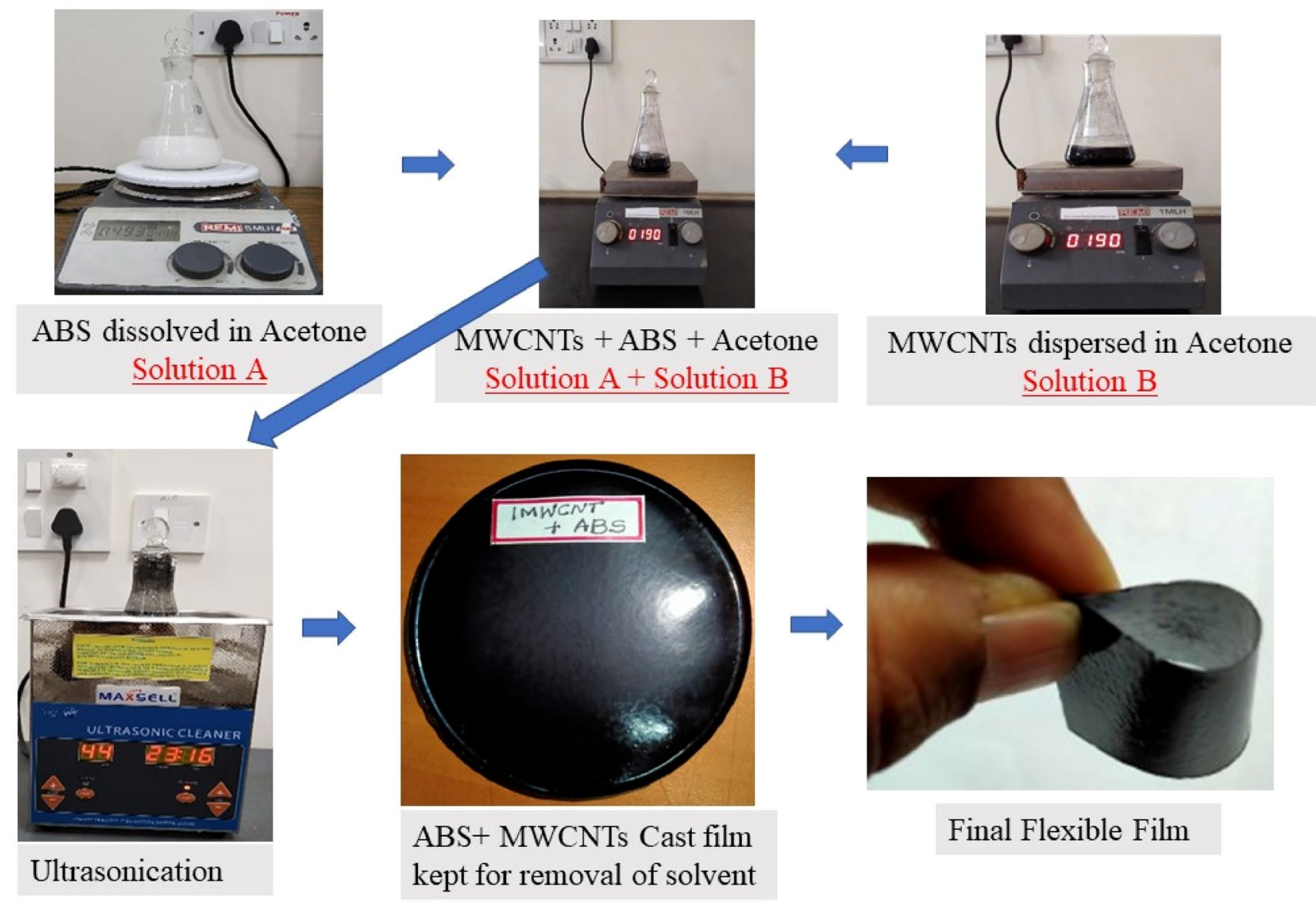

Fig. 1 Fabrication process of flexible MWCNT-ABS films 


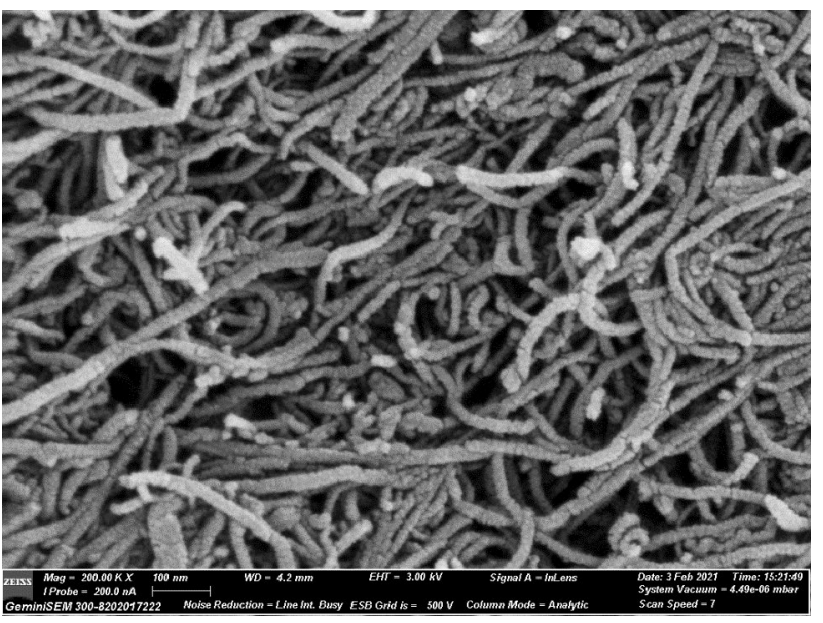

Fig. 2 FESEM image of MWCNTs

method and also compounding and melt-mixing methods. The functionalization of CNTs may facilitate uniform dispersion in the matrix and enhance mechanical properties due to efficient load transfer between filler and matrices. However, it is reducing the conductivity of the composite. Also, the compounding and extrusion method involves two heat cycles. Due to more number heat cycle while manufacturing reduces the final product's thermal stability, and this process also generates much waste. From this point of view, we attempted to develop thin films by the slow speed solution mixing method to avoid breakage of MWCNTs and achieve a better dispersion of non-functionalized low-quantity MWCNTs in ABS. The tensile properties of neat-ABS films and various wt\% MWCNT-ABS nanocomposite films are studied using a tensile testing machine at the strain rate of $0.02 \mathrm{~S}^{-1}$. Five samples were tested in each type of MWCNT-ABS nanocomposite and neat-ABS films. The average values are considered. The stress-strain behavior is plotted of all the films as shown in Fig. 3a. The Young's modulus and tensile strength of neat-ABS and MWCNT-ABS nanocomposite films are shown in Fig. $3 b$.

The Young's modulus has increased with an increase in the content of MWCNTs in composite films. Highest Young's modulus noticed for $1 \mathrm{wt} \%$ MWCNT-ABS composite film. The tensile strength is increased due to the addition of $0.25 \mathrm{wt} \%$ MWCNTs in the composites. It shows the highest tensile strength and Young's modulus as compared to all the composite films. Further addition of MWCNTs decreased the tensile strength, and it is lesser than neat-ABS at 1 wt. \% MWCNT content. This trend is because, at higher concentrations, non-uniform dispersion and agglomeration occur due to high aspect ratio MWCNTs. However, the $0.5 \mathrm{wt} \%$ MWCNT-ABS composites show better mechanical properties compared to neat-ABS composites.
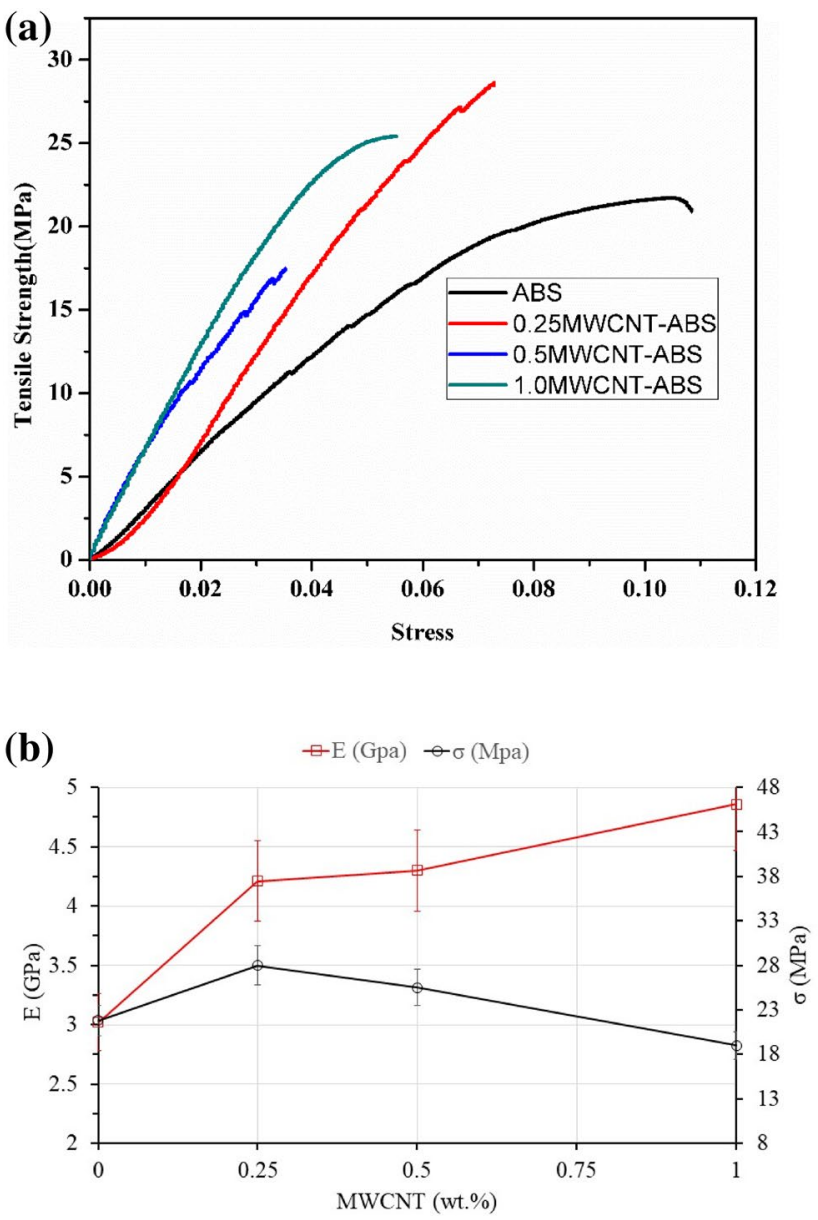

Fig. 3 a Stress-strain plot of neat-ABS and MWCNT-ABS composite films. b Tensile strength and Young's modulus of neat-ABS and MWCNT-ABS nanocomposite films

\subsection{Thermal Analysis}

Thermal degradation stability of the neat-ABS and MWCNT-ABS nanocomposite films were studied by thermogravimetric analysis in the inert atmosphere with a $10{ }^{\circ} \mathrm{C} /$ min heating rate. The TGA curves of neat-ABS films and MWCNT-ABS nanocomposite films were plotted, as shown in Fig. 4. The observed weight loss in the neat-ABS between the temperature range of $127-130{ }^{\circ} \mathrm{C}$ is due to the evaporation of solvent content. Whereas MWCNT-ABS nanocomposite indicates a plateau, and there is no degradation of samples in this range. The weight losses of neat-ABS and ABS-MWCNTs nanocomposites are due to the polymer chain's breakdown, oxidation of organic groups, and evaporation of dopants starts in the range $370-470{ }^{\circ} \mathrm{C}$. The addition of MWCNTs in ABS mitigates the mass transfer, leading to an interruption in weight loss. All the MWCNTABS nanocomposite films show a marginal weight loss at a predicted temperature and show overall improved thermal stability compared to the neat-ABS film sample. The weight 
(a)

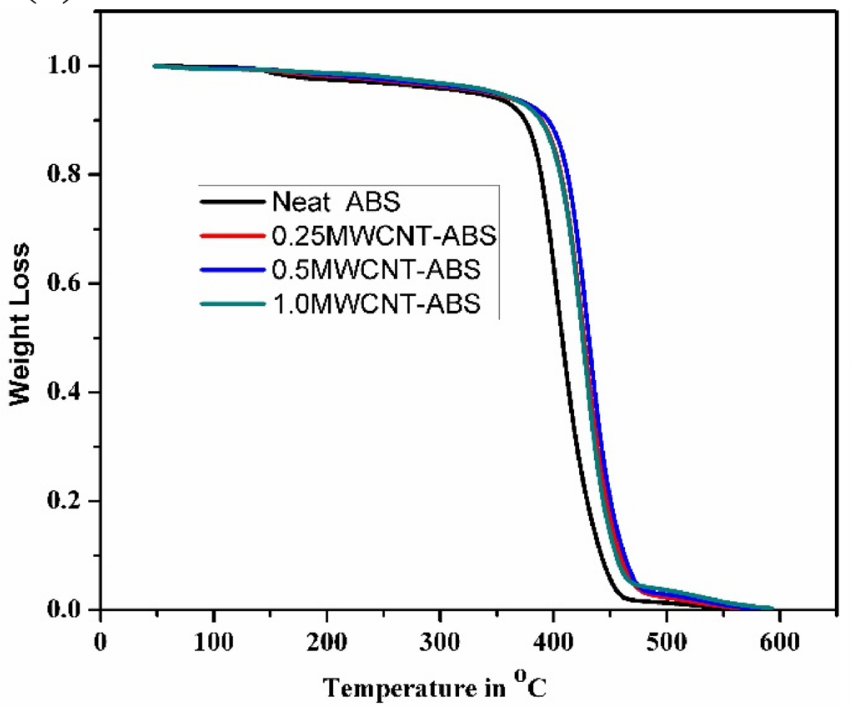

(b)

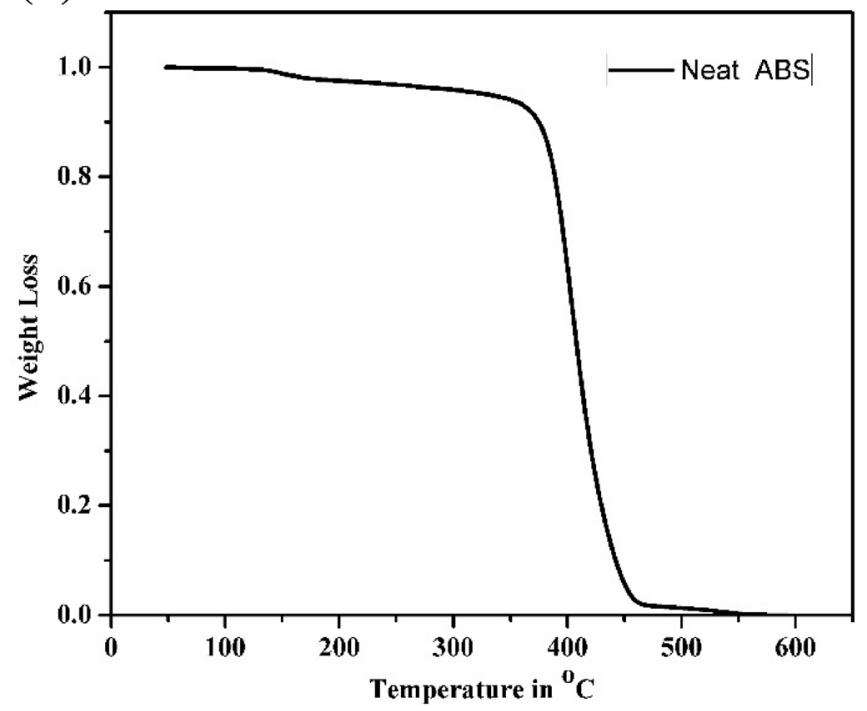

(c)

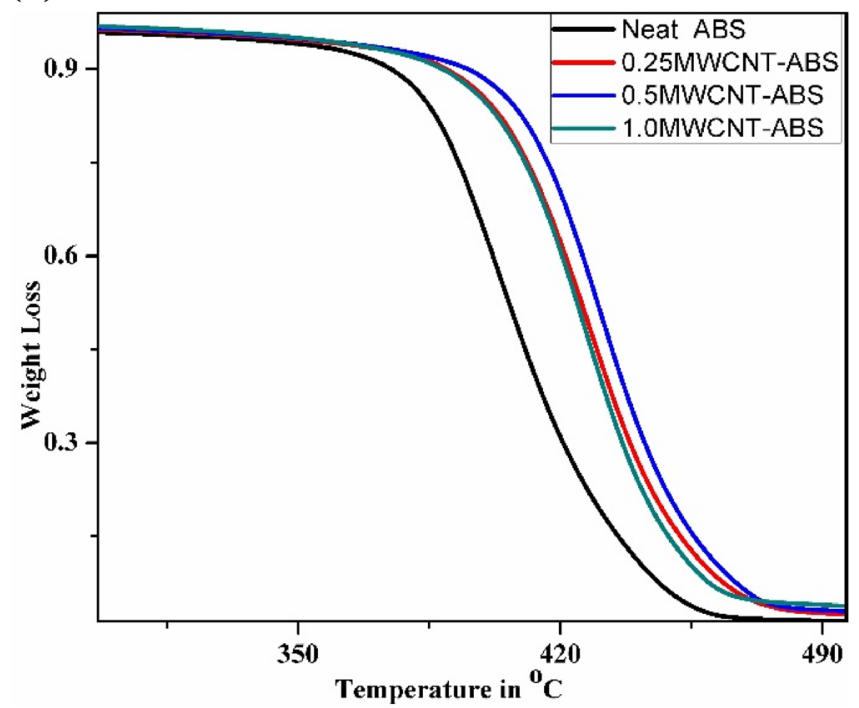

Fig. 4 Thermo-gravimetric curves a Neat-ABS and MWCNT-ABS nanocomposites, $\mathbf{b}$ enlarged TG curve of neat-ABS, and $\mathbf{c}$ enlarged TG curves for neat-ABS and MWCNT-ABS nanocomposites

Table 2 Thermal degradation characteristics of neat-ABS and MWCNT-ABS composite films

\begin{tabular}{llllll}
\hline Films & $\begin{array}{l}\text { MWCNTs in } \\
\text { ABS }(w t \%)\end{array}$ & $\begin{array}{l}20 \% \text { wt. loss at } \\
\text { temperature }\left({ }^{\circ} \mathrm{C}\right)\end{array}$ & $\begin{array}{l}50 \% \text { wt. loss at } \\
\text { temperature }\left({ }^{\circ} \mathrm{C}\right)\end{array}$ & $\begin{array}{l}\text { Improvement in wt. } \\
\text { loss temperature } \\
\left({ }^{\circ} \mathrm{C}\right)\end{array}$ \\
\cline { 3 - 6 } & & 430 & & $-20 \%$ & @ 50\% \\
\hline Neat ABS & 0 & 447 & 407 & - & - \\
MWCNT-ABS & 0.25 & 450 & 427 & 20 & 24 \\
& 0.5 & 444 & 431 & 14 & 18 \\
\hline
\end{tabular}


loss of ABS and MWCNT-ABS nanocomposites are shown in Table 2. From the data, one can see the enhancement of thermal degradation temperature 17 and $20{ }^{\circ} \mathrm{C}$, and 20 and $24{ }^{\circ} \mathrm{C}$ for the nanocomposites over neat-ABS due to the addition of $0.25 \mathrm{wt} \%$ and $0.5 \mathrm{wt} \%$ MWCNTs, respectively. The $0.25 \mathrm{wt} \%$ and $0.5 \mathrm{wt} \%$ MWCNT-ABS nanocomposite shows a better result in $20 \%$ and $50 \%$ weight loss. However, the $1 \mathrm{wt} \%$ MWCNT-ABS nanocomposite shows marginal improvement in the thermal degradation compared to neatABS because of the inconsistency of content of filler in the sample due to agglomeration.

\subsection{Electrical Conductivity}

The most widely used electrical conductivity measurement technique for polymer nanocomposite films is the four-probe conductivity measurement method as per ASTM D-257. In the present study, five identical square shapes of $5 \times 5 \mathrm{~mm}$ samples were prepared by each MWCNT-ABS composite films. The thin copper wires are attached to the samples with the help of high purity silver paste. A constant DC source (Keithley 6221) is used to deliver a one mA current passing through the sample by two outer probes. The two inner probes are used to measure the voltage using a nano-voltmeter (Model: 2182-A, Keithley). The conductivity of each sample was recorded, and the average value of the electrical conductivity versus wt\% of MWCNTs content is plotted, as shown in Fig. 5.

The MWCNT-ABS nanocomposite films consist of MWCNTs up to $0.25 \mathrm{wt} \%$ exhibit a considerable increase in the DC electrical conductivity, as depicted in Fig. 5. The resistivity of the pristine ABS sample $\left(1.3 \times 10^{15} \Omega\right.$-cm) decreased to $\left(7.98 \times 10^{6} \Omega\right.$-cm) when MWCNTs content was increased to $1.0 \mathrm{wt} \%$. The decrease in electrical resistivity ( $\sim 10^{9}$ order of magnitude) signifies the formation of the network structure of the MWCNTs in the ABS matrix. Also, quantum effects such as electronic tunneling between the neighboring MWCNTs in the ABS matrix increased

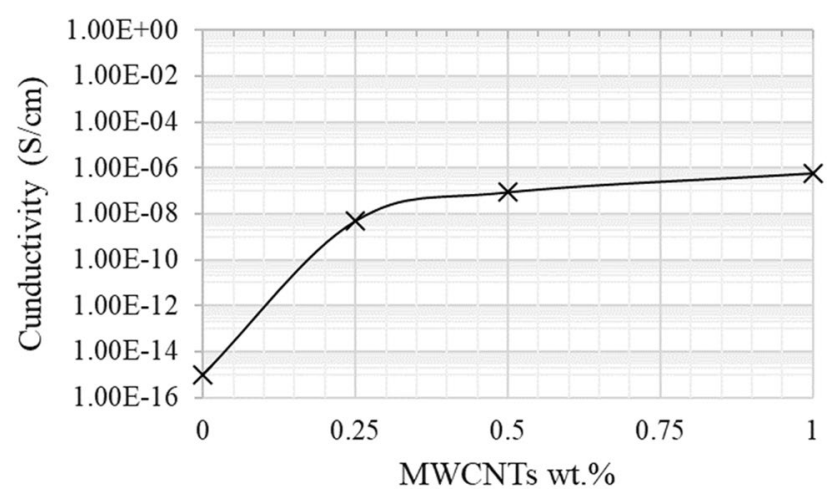

Fig. 5 Plot of electrical conductivity of MWCNT-ABS films the conductivity. Due to the high electrical conductivity of MWCNTs, the interconnected network structures developed a conductive path in low concentration $(0.25 \mathrm{wt} \%)$ of MWCNT-ABS nanocomposites, which is due to the electrical percolation phenomenon. The percolation concentration and the percolation behavior depend on many factors, including the dispersion, distribution, and aspect ratio of the conductive filler [9]. In the melt-mixing method, the chance of breaking the high aspect ratio multiwalled nanotube due to a higher required shear rate while mixing. In the present research work, since we are used the solution mixing method, the aspect ratio of MWCNTs has a considerable influence on the nanocomposites' conductivity. Hence, we are getting at a lower percolation concentration. However, it is found that the conductivity is saturated at 0.5 and $1 \mathrm{wt} \%$ of MWCNTs concentrations. This saturation is because at higher quantity mixing (sonication process), increased the chances of collisions of MWCNTs. This enhancement causes degradation of aspect ratio owing to the negative effect on networking structure. Further, it leads to an agglomeration of broken MWCNTs in the ABS matrix.

\subsection{Electromagnetic Shielding Effectiveness}

EMSE is the capability of the shielding material to attenuate electromagnetic waves. A vector network analyzer with a waveguide flange type adopter is used to acquire $\mathrm{S}$ parameters in the present work. Two-port calibration was performed without a sample to subtract the free space loss. In this work, we selected an X-band frequency range- $8 \mathrm{GHz}$ to $12 \mathrm{GHz}$ to investigate the EMSE. The rectangular shape samples with a dimension of $2.286 \times 1.016 \mathrm{~cm}$ were prepared from neat-ABS and in each type of MWCNT-ABS nanocomposite films. The sample is kept in the sample holder while performing the test. The test setup is shown in Fig. 6. The EMSE is composed of reflection loss, absorption loss, and multiple reflection loss. However, the multiple reflection loss is not considered because it is not significant in the highfrequency range. The shielding effectiveness (SE) reflection loss $\mathrm{SE}_{\mathrm{RL}}$ and absorption losses $\mathrm{SE}_{\mathrm{AL}}$ is calculated from measured scattering parameters $S_{11}$ and $S_{21}$ by the following formulas:

EMSE $=10 \log \left[\frac{1}{\left|S_{12}\right|^{2}}\right]=10 \log \left[\frac{1}{\left|S_{21}\right|^{2}}\right]$

In the above equation, the power transmitted from port $i$ to port $j$ is represented by $\left|S_{I J}\right|^{2}$. In the absence of any shielding, the incident power equals transmitting power, i.e., $\left|S_{I J}\right|^{2}=1.0$. The reflected, transmitted, and absorption powers $(\mathrm{R}, \mathrm{T}$, and $\mathrm{A})$ and the absorption and reflection losses $\left(\mathrm{SE}_{\mathrm{A}}, \mathrm{SE}_{\mathrm{R}}\right)$, are calculated using Eqs. 2-6 with $\mathrm{S}$ parameters 


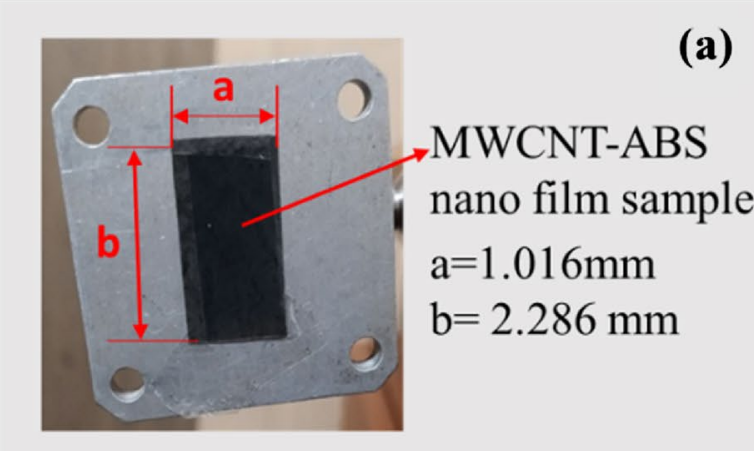

\section{Waveguide specimen adopter}
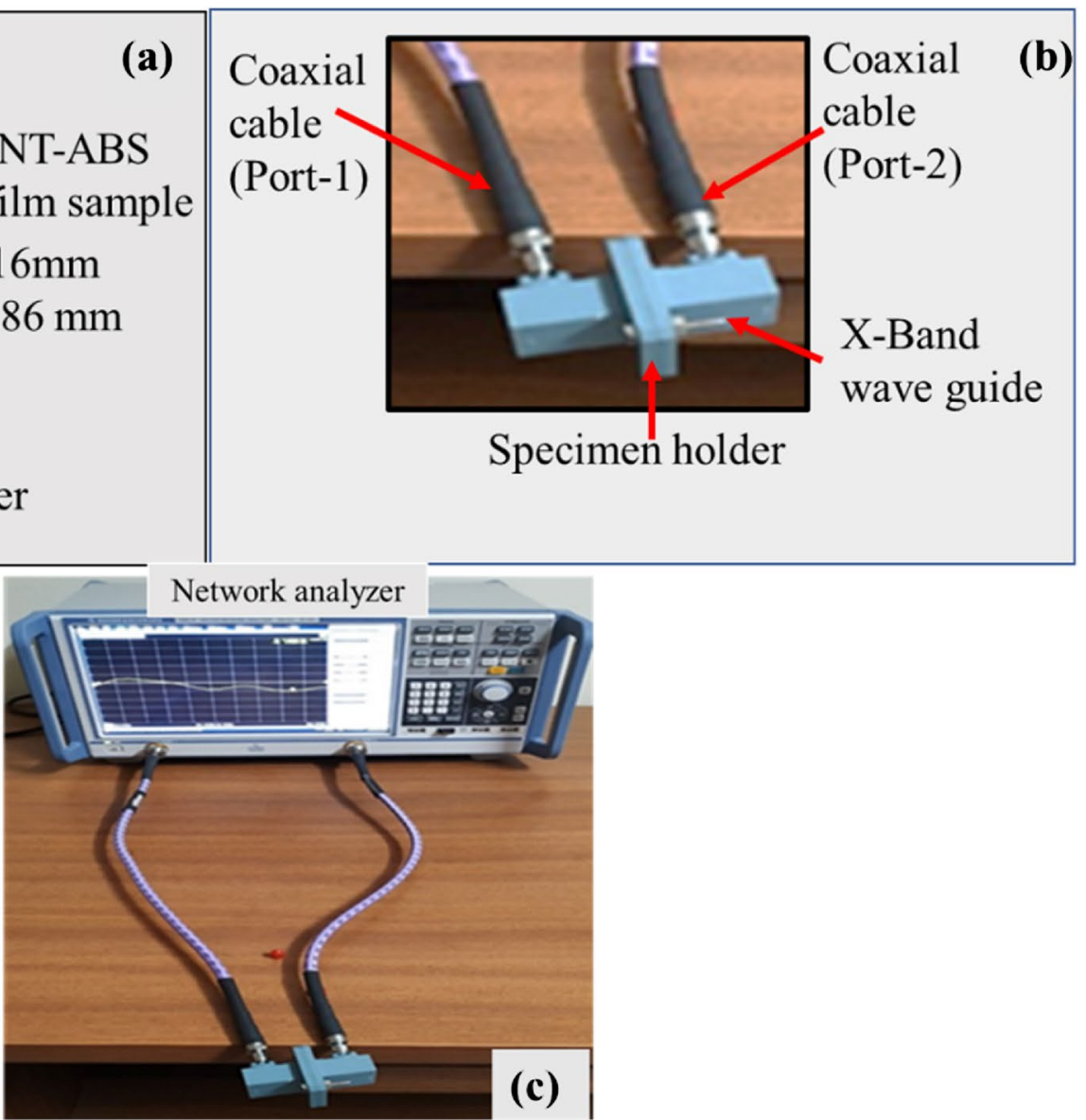

Fig. 6 a Sample holder containing specimens, b X-band waveguide assembly with a sample, and c network analyzer connected with coaxial cables

obtained from the experiments. Finally, the total SE is calculated by Eq. 7 .

$\mathrm{R}=\left|\mathrm{S}_{22}\right|^{2}=\left|\mathrm{S}_{11}\right|^{2}$

$\mathrm{T}=\left|\mathrm{S}_{21}\right|^{2}=\left|\mathrm{S}_{12}\right|^{2}$

$\mathrm{A}=1-\mathrm{R}-\mathrm{T}$

$\mathrm{SE}_{\mathrm{AL}}=10 \log \left(1-\left|\mathrm{S}_{22}\right|^{2} /\left|\mathrm{S}_{21}\right|^{2}\right)$

$\mathrm{SE}_{\mathrm{RL}}=10 \log \left(1 /\left(1-\left|\mathrm{S}_{11}\right|^{2}\right)\right)$

$\mathrm{SE}_{\mathrm{TL}}=\mathrm{SE}_{\mathrm{RL}}+\mathrm{SE}_{\mathrm{AL}}$

The absorption, reflection, and SE of neat-ABS and MWCNT-ABS nanocomposite films are shown in Fig. 7. The EMSE of the studied samples at $9 \mathrm{GHz}$ is shown in Table 3.
It is evident that at $0.5 \mathrm{wt} \%$ ABS-MWCNT nanocomposite exhibits SE of $15.07 \mathrm{~dB}$ in the X-band, further increases in the loading percentage of MWCNT decreased in the SE to $12.06 \mathrm{~dB}$.

Figure 7d shows the schematic representation of MWCNTs dispersion in the ABS matrix and found in the experimentation that the EMSE increased due to the addition of MWCNTs. This because of the networking structure of MWCNTs due to their high aspect ratio developed in the ABS matrix. The mechanism of attenuation of EM waves in the material takes place due to absorption and reflection. The reflection is more as compared to absorption in all the results. It is due to the concentration of MWCNTs on the surface of the film that makes it more conductive. This phenomenon helps to develop multilayer composites with ABS-MWCNT nanofilms. 


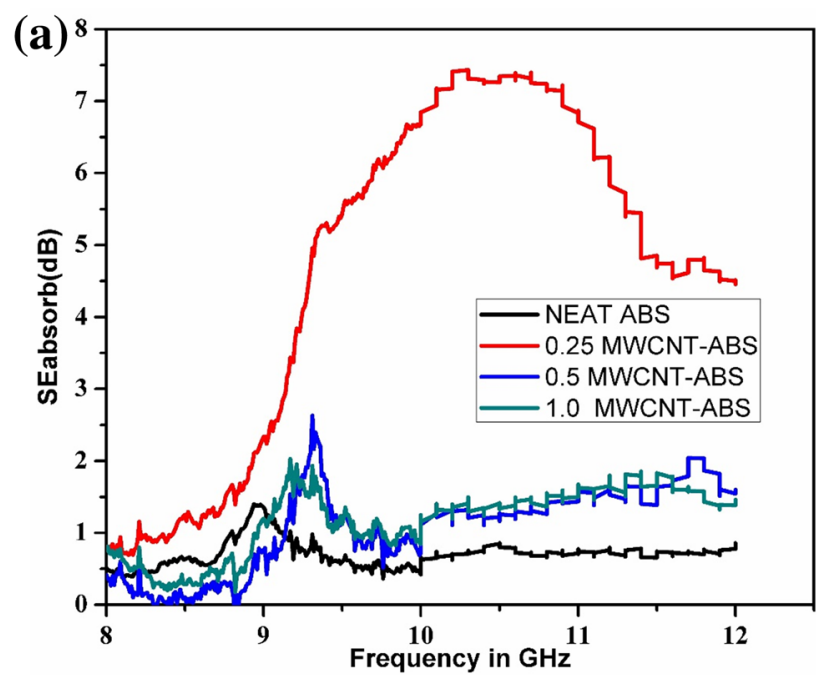

(b)

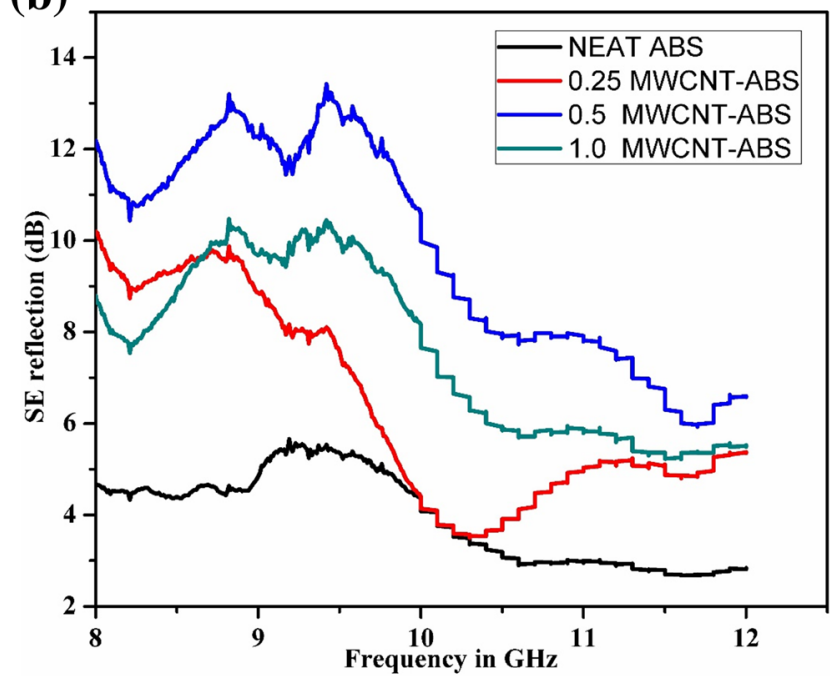

Fig. 7 a EMSE due to absorption. b EMSE due to reflection in $\mathrm{X}$-band frequency range. c EMSE of neat-ABS and MWCNT-ABS nanocomposite films. d Schematic representation of dispersed MWCNTs in the ABS matrix

\section{Conclusions}

Microfilms were fabricated by MWCNTs dispersed in ABS polymer by solution method followed hot compression. It was observed that the MWCNTs are dispersed uniformly at low concentrations $(0.25 \mathrm{wt} \%)$ and agglomerated at higher concentrations ( $1 \mathrm{wt} \%)$. The mechanical properties such as tensile strength and modulus of ABS films improved by adding $0.25 \mathrm{wt} \%$ of MWCNTs. Further, we found that $0.25 \mathrm{wt} \%$ MWCNT-ABS composite films show better electrical conductivity and EMSE compared to neat-ABS composite films. However, the $0.5 \mathrm{wt} \%$

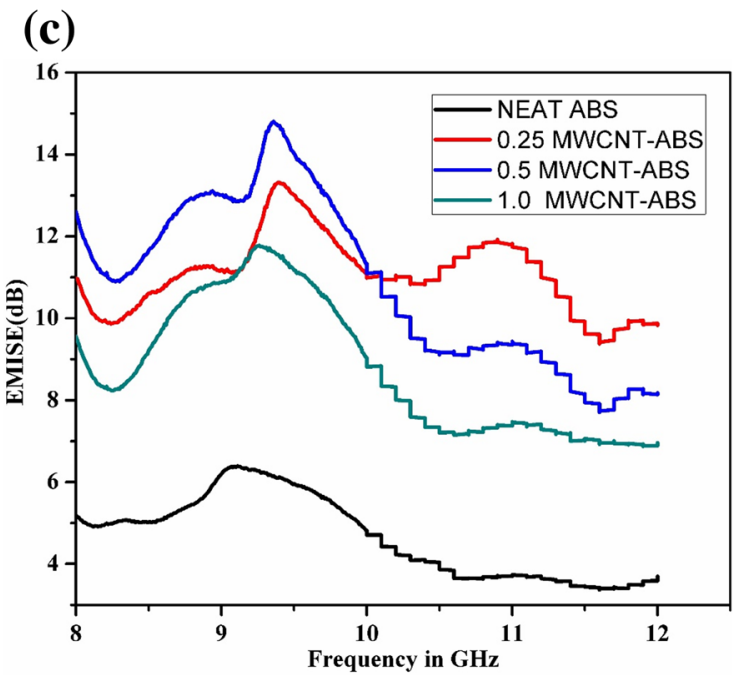

(d)

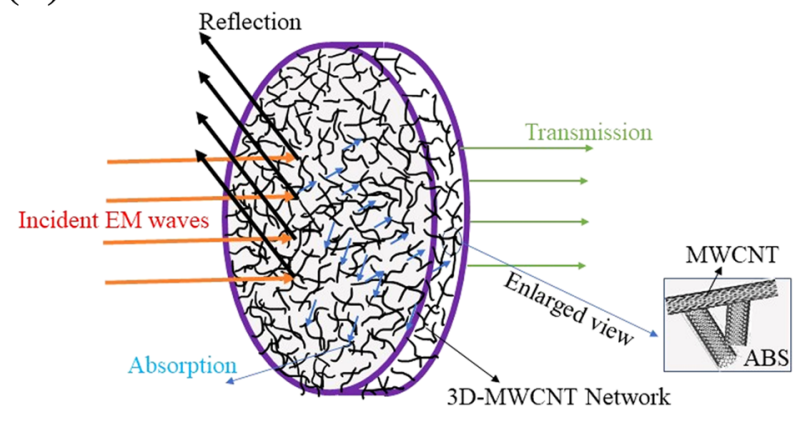

Fig. 7 (continued)

Table 3 The EMSE of neat-ABS and MWCNT-ABS nanocomposite films

\begin{tabular}{llcc}
\hline Sample code & $\mathrm{SE}_{\mathrm{A}}(\mathrm{dB})$ & $\mathrm{SE}_{\mathrm{R}}(\mathrm{dB})$ & $\mathrm{SE}_{\mathrm{T}}(\mathrm{dB})$ \\
\hline ABS & 1.12 & 5.2 & 6.32 \\
0.25MWCNT-ABS & 2.5 & 7.9 & 10.4 \\
0.5MWCNT-ABS & 2.17 & 12.9 & 15.07 \\
1.0MWCNT-ABS & 1.9 & 10.16 & 12.06 \\
\hline
\end{tabular}

MWCNT-ABS composites show better EMSE compared to all the samples. Hence, we recommend investigating the composite made of multiple layers of these films to develop nanocomposites suitable for X-band electromagnetic shielding applications. The content of MWCNTs in polymer nanocomposite decides the economic viability of practical applications as its cost is higher. In this work, we use smaller quantities of MWCNTs and a simple fabricating method without much energy required for processing. The films can be utilized better to protect the composite as the content of MWCNTs in polymer composites is critical. 
Funding Open access funding provided by Manipal Academy of Higher Education, Manipal.

Open Access This article is licensed under a Creative Commons Attribution 4.0 International License, which permits use, sharing, adaptation, distribution and reproduction in any medium or format, as long as you give appropriate credit to the original author(s) and the source, provide a link to the Creative Commons licence, and indicate if changes were made. The images or other third party material in this article are included in the article's Creative Commons licence, unless indicated otherwise in a credit line to the material. If material is not included in the article's Creative Commons licence and your intended use is not permitted by statutory regulation or exceeds the permitted use, you will need to obtain permission directly from the copyright holder. To view a copy of this licence, visit http://creativecommons.org/licenses/by/4.0/.

\section{References}

1. J. Wang, H. Zhou, J. Zhuang, Q. Liu, Influence of spatial configurations on electromagnetic interference shielding of ordered mesoporous carbon/ordered mesoporous silica/silica composites. Sci. Rep. 3, 3252 (2013)

2. Y. Huang, N. Li, Y. Ma, F. Du, F. Li, X. He, X. Lin, H. Gao, Y. Chen, The influence of single-walled carbon nanotube structure on the electromagnetic interference shielding efficiency of its epoxy composites. Carbon 45, 1614-1621 (2007)

3. D.D.L. Chung, Electromagnetic interference shielding effectiveness of carbon materials. Carbon 39, 279-285 (2001)

4. D.D.L. Chung, Carbon materials for structural self-sensing, electromagnetic shielding and thermal interfacing. Carbon 50, 3342-3353 (2012)

5. D. Micheli, C. Apollo, R. Pastore, R.B. Morles, S. Laurenzi, M. Marchetti, Nanostructured composite materials for electromagnetic interference shielding applications. Acta Astronaut. 69, 747-757 (2011)

6. P.C.P. Watts, W.K. Hsu, A. Barnes, B. Chambers, High permittivity from defective multiwalled carbon nanotubes in the X-band. Adv Mater. 15(7-8), 600-603 (2003)

7. D.P. Schmitz, L.G. Ecco, S. Dul, E.C.L. Pereira, B.G. Soares, G.M.O. Barra, A. Pegoretti, Electromagnetic interference shielding effectiveness of ABS carbon-based composites manufactured via fused deposition modelling. Mater. Today Commun. 15, 70-80 (2018)

8. D.P. Schmitz, T.I. Silva, S.D.A.S. Ramoa, G.M.O. Barra, A. Pegoretti, B.G. Soares, Hybrid composites of ABS with carbonaceous fillers for electromagnetic shielding applications. J. Appl. Polym. Sci. 46546, 1-10 (2018)

9. M.H. Al-Saleh, U. Sundararaj, Electromagnetic interference shielding mechanisms of CNT/polymer composites. Carbon $\mathbf{4 7}$, 1738-1746 (2009)

10. U. Basuli, S. Chattopadhyay, C. Nah, T.K. Chaki, Electrical properties and electromagnetic interference shielding effectiveness of multiwalled carbon nanotubes-reinforced EMA nanocomposites. Polym. Compos. 33(6), 897-903 (2012)

11. W.S. Jou, H.Z. Cheng, C.F. Hsu, The electromagnetic shielding effectiveness of carbon nanotubes polymer composites. J. Alloys Compd. 434-435, 641-645 (2007)

12. D. Micheli, C. Apollo, R. Pastore, D. Barbera, R.B. Morles, M. Marchetti et al., Optimization of multilayer shields made of composite nanostructured materials. IEEE Trans. Electromagn. Compat. 54, 60-69 (2012)

13. C.F. Kuan, K.C. Lin, C.L. Chiang, C.H. Chen, H.C. Peng, H.C. Kuan, Effect of modification method and processing condition on the properties of multiwall carbon nanotube/acrylonitrile-butadiene-styrene nanocomposite. Adv. Sci. Lett. 19(2), 559-561 (2013)

14. M.H. Al-Saleh, U. Sundararaj, Microstructure, electrical, and electromagnetic interference shielding properties of carbon nanotube/ acrylonitrile-butadiene-styrene nanocomposites. J. Polymer. Sci. 50, 1356-1362 (2012)

15. M.H. Al-Saleh, Influence of conductive network structure on the EMI shielding and electrical percolation of carbon nanotube/polymer nanocomposites. Synth. Met. 205, 78-84 (2015)

16. J. Jyoti, S. Basu, B.P. Singh, S.R. Dhakate, Superior mechanical and electrical properties of multiwall carbon nanotube reinforced acrylonitrile-butadiene-styrene high performance composites. Compos. B Eng. 83, 58-65 (2015)

17. S. Kapoor, M. Goyal, P. Jindal, Enhanced thermal, static, and dynamic mechanical properties of multi-walled carbon nanotubes-reinforced acrylonitrile-butadiene-styrene nanocomposite. J. Thermoplast. Compos. Mater. (2019). https://doi.org/10.1177/ 0892705719886012

18. ASTM D882-18, Standard test method for tensile properties of thin plastic sheeting, ASTM International, West Conshohocken, PA, 2018. www.astm.org

19. ASTM D257-14, Standard test methods for DC resistance or conductance of insulating materials, ASTM International, West Conshohocken, PA, 2014. www.astm.org

Publisher's Note Springer Nature remains neutral with regard to jurisdictional claims in published maps and institutional affiliations. 